Check for updates

Cite this: Phys. Chem. Chem. Phys., 2020, 22, 10426

Received 2nd December 2019, Accepted 19th December 2019

DOI: $10.1039 / c 9 c p 06479 f$

rsc.li/pccp

\section{Temperature effects on the ionic conductivity in concentrated alkaline electrolyte solutions $\dagger$}

\author{
Yunqi Shao, (D) a Matti Hellström, (D) bc Are Yllö, ${ }^{a}$ Jonas Mindemark, (D) a \\ Kersti Hermansson, (D) a Jörg Behler (D) ${ }^{\mathrm{b}}$ and Chao Zhang (D) *a
}

\begin{abstract}
Alkaline electrolyte solutions are important components in rechargeable batteries and alkaline fuel cells. As the ionic conductivity is thought to be a limiting factor in the performance of these devices, which are often operated at elevated temperatures, its temperature dependence is of significant interest. Here we use $\mathrm{NaOH}$ as a prototypical example of alkaline electrolytes, and for this system we have carried out reactive molecular dynamics simulations with an experimentally verified high-dimensional neural network potential derived from density-functional theory calculations. It is found that in concentrated $\mathrm{NaOH}$ solutions elevated temperatures enhance both the contributions of proton transfer to the ionic conductivity and deviations from the Nernst-Einstein relation. These findings are expected to be of practical relevance for electrochemical devices based on alkaline electrolyte solutions.
\end{abstract}

Because of their excellent ionic conductivity and high roomtemperature solubility, alkaline electrolyte solutions are widely used in electrochemical devices such as rechargeable batteries and alkaline fuel cells. ${ }^{1,2}$ The electrochemically active ion in alkaline electrolytes is the hydroxide ion. ${ }^{3} \mathrm{OH}^{-}$has an anomalously high mobility in aqueous solution, as it can diffuse via Grotthuss mechanism which is composed of a series of proton transfer events. ${ }^{4}$ Major progress in the understanding of $\mathrm{OH}^{-}$ solvation and mobility at low concentration was made by molecular dynamics (MD) simulations based on density functional theory $^{5-9}$ and reactive force fields, ${ }^{10,11}$ which highlighted the importance of "presolvation", i.e., a thermally induced hydrogenbond fluctuation, in the diffusion of hydroxide ions. ${ }^{7,10-12}$

Although ionic conductivity at low concentrations is welldescribed by the Nernst-Einstein equation, which links the

\footnotetext{
${ }^{a}$ Department of Chemistry - Ångström Laboratory, Uppsala University, Box 538, 75121 Uppsala, Sweden. E-mail: chao.zhang@kemi.uu.se

${ }^{b}$ Universität Göttingen, Institut für Physikalische Chemie, Theoretische Chemie, Tammannstr. 6, 37077 Göttingen, Germany

${ }^{c}$ Software for Chemistry and Materials B. V., De Boelelaan 1083, 1081 HV Amsterdam, The Netherlands

$\dagger$ Electronic supplementary information (ESI) available. See DOI: 10.1039/ c9cp06479f
}

conductivity $\sigma$ to the self-diffusion coefficients $D_{+}$and $D_{-}$of cations and anions, respectively, this simple picture is no longer valid at concentrations typically used in electrochemical devices. At higher concentrations, several types of non-ideal phenomena like ion-pairing, ${ }^{13}$ in which cations and anions associate and form metastable neutral pairs, and, more generally, cross-correlations of the movements of different ions (of equal or opposite charge) can notably alter the ionic conductivity. Moreover, the working temperature of alkaline batteries and fuel cells can be much higher than room-temperature $(293 \mathrm{~K}) .{ }^{14}$ Therefore, it is desirable to understand the temperature effects on proton transfer and ion-pairing in alkaline electrolyte solutions and their implications concerning the ionic conductivity at high concentrations and elevated temperatures.

Simulations of electric properties, such as ionic conductivity, necessitate long time-scales and, except for cases at extreme conditions, ${ }^{15}$ are normally beyond reach of the standard densityfunctional theory (DFT)-based MD. One way to tackle this timescale challenge is to explore finite-field DFTMD simulations to speed up the convergence of the polarization $\mathbf{P}$, which has been successfully applied to compute the dielectric constant of polar liquids and the capacitance of electrified solid-electrolyte interfaces. ${ }^{16}$ The other approach to solve this problem is to make use of reactive force fields to access longer timescales. ${ }^{10,11,17}$ One promising approach in this direction is to devise high-dimensional neural network potentials (NNPs) with DFT quality as proposed by Behler and Parrinello. ${ }^{18}$ Here, we use this approach, and by means of MD simulations using a NNP for the prototypical case of aqueous $\mathrm{NaOH}$ solutions ${ }^{19}$ and show how different factors together lead to the surprising behavior of the ionic conductivity in concentrated $\mathrm{NaOH}$ aqueous solutions at elevated temperatures.

The details of the construction and validation of the NNP for $\mathrm{NaOH}$ solutions using DFT calculations at the dispersioncorrected GGA level have previously been discussed in detail ${ }^{19-21}$ (see also a brief summary in ESI $\dagger$ ). The present MD simulations were performed using LAMMPS ${ }^{22}$ together with an extension for high-dimensional NNPs. ${ }^{23}$ The cubic simulation box contained 
between 272 and 496 water molecules, and between 8 and 120 $\mathrm{NaOH}$ formula units, depending on concentration (see Table S1 in the ESI $\dagger$ ). The length of cubic simulation box has been fixed using the experimental densities of $\mathrm{NaOH}$ solutions at the given composition and temperature $^{24}$ (see Table S1 in the ESI $\dagger$ ). Production runs with a timestep of 0.5 fs in the NVT ensemble lasted for $15 \mathrm{~ns}$ at each combination of composition and temperature after the equilibration. The Bussi-Donadio-Parrinello thermostat $^{25}$ which has shown an excellent control of kinetic energy and little effect on the dynamical properties was employed. The trajectory frames were saved every 0.01 ps for later analysis. Each trajectory was split into 5 uncorrelated segments with length of $3 \mathrm{~ns}$ each. The standard deviations of observables from the different segments were used as an error estimate. Note that nuclear quantum effects were not included in the MD simulations.

To accompany the simulations, we have also performed conductivity measurements of $\mathrm{NaOH}$ solutions of concentrations up to 25 molality $(\mathrm{m})$. The conductivity meter probe used is a 4 pole InLab 738-ISM by (Mettler Toledo) which has a sensitivity range from $0.01-1000 \mathrm{mS} \mathrm{cm} \mathrm{cm}^{-1}$ and gives accurate measurements up to $373 \mathrm{~K}$. The mean and the standard deviation of five independent measurements after calibration were reported for each given $\mathrm{NaOH}$ solution at both $293 \mathrm{~K}$ and $323 \mathrm{~K}$.

When comparing simulation and experimental results, it is important to realize that the ionic conductivity can be computed using different formulas which have different applicabilities. As mentioned at the beginning, the Nernst-Einstein equation for the ionic conductivity of a 1:1 symmetric electrolyte is valid only at low concentration and can be written as

$$
\sigma_{\mathrm{N}-\mathrm{E}}=q^{2} \rho \beta\left(D_{+}+D_{-}\right),
$$

where $\beta$ is the inverse temperature, $q$ is the formal charge of each ion and $\rho$ is the number density of the formula unit of the $1: 1$ electrolyte.

$D_{+}$can be obtained by integrating the velocity auto-correlation function as

$$
D_{+}=\frac{1}{3} \int_{0}^{\infty} \mathrm{d} t\left\langle\mathbf{v}_{i,+}(0) \mathbf{v}_{i,+}(t)\right\rangle
$$

where $t$ is time and $\mathbf{v}_{i,+}$ is the velocity vector of the $i$ th cation, and the average is taken over all cations and time origins. Alternatively, the Einstein relation

$$
D_{+}=\lim _{t \rightarrow \infty} \frac{1}{6 t}\left\langle\left[\mathbf{r}_{i,+}(t)-\mathbf{r}_{i,+}(0)\right]^{2}\right\rangle
$$

can be used, where $\mathbf{r}_{i,+}$ is the position of the $i$ th cation.

$D_{-}$can be computed analogously according to eqn (2) and (3). We defined the positions of $\mathrm{OH}^{-}$ions by the position of $\mathrm{O}$ atoms bonded only to a single $\mathrm{H}$ atom. All bonds in the system were defined by assigning each hydrogen atom to its nearest oxygen atom, which gave either water molecules or hydroxide ions. Upon proton transfer reactions, the trajectories of $\mathrm{OH}^{-}$were traced in a fashion similar to how was done in ref. 21, i.e. based on "the Hungarian algorithm" as introduced by König in 1916 and Egerváry in 1931 and elaborated by Kuhn in $1955 .^{26}$

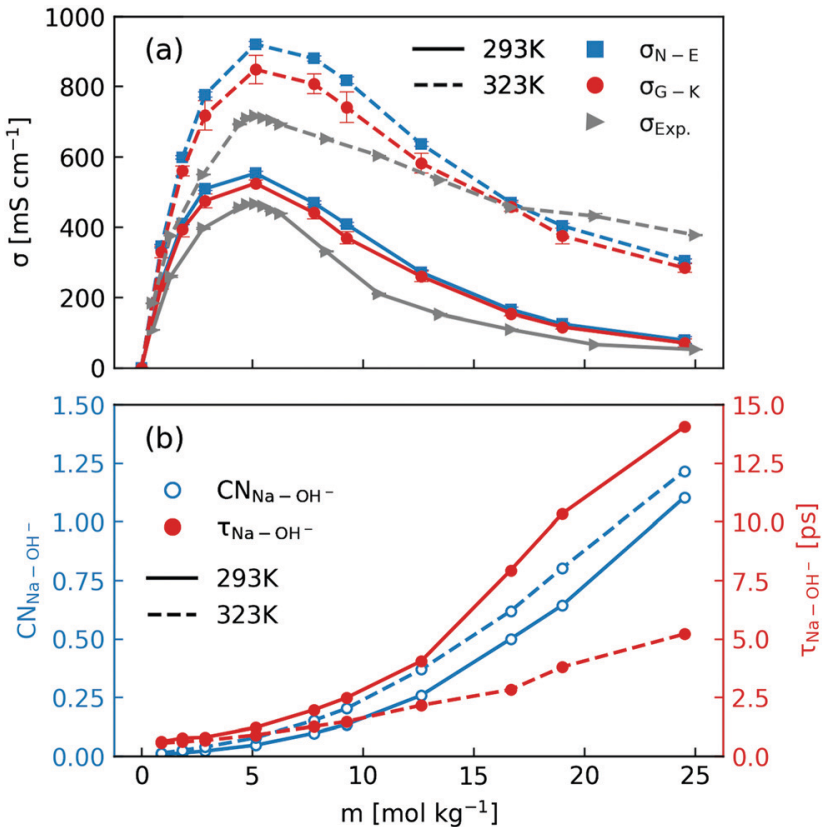

Fig. 1 (a) Comparison of concentration-dependent ionic conductivities calculated using the Nernst-Einstein formula (eqn (1) and (3)) and the Green-Kubo formula (eqn (5)) from MD simulations and those measured in experiments at $293 \mathrm{~K}$ and $323 \mathrm{~K}$; (b) calculated $\mathrm{Na}^{+}-\mathrm{OH}^{-}$coordination numbers and residence time.

The Nernst-Einstein equation becomes approximate at high concentrations, for which ion-pairing and cross-correlated ion motions play an important role. ${ }^{27-29}$ A more general equation for the conductivity, which is valid at both low and high concentrations, is the Green-Kubo formula

$$
\sigma_{\mathrm{G}-\mathrm{K}}=\frac{\beta}{3} \int \mathrm{d} \mathbf{r} \int_{0}^{\infty} \mathrm{d} t\langle\mathbf{J}(0,0) \mathbf{J}(\mathbf{r}, t)\rangle
$$

where $\mathbf{J}$ is the current density. Alternatively, the equivalent Einstein-type relation can also be used ${ }^{30}$

$$
\sigma_{\mathrm{G}-\mathrm{K}}=\lim _{t \rightarrow \infty} \frac{\beta \Omega}{6 t}\left\langle[\mathbf{P}(t)-\mathbf{P}(0)]^{2}\right\rangle
$$

where $\mathbf{P}$ is the itinerant polarization in ionic solution ${ }^{31}$ and $\Omega$ is the volume of the simulation box.

Unlike $\sigma_{\mathrm{N}-\mathrm{E}}, \sigma_{\mathrm{G}-\mathrm{K}}$ includes ion-pairing and cross-correlated ion motions from the so-called distinct diffusion coefficients of cations $D_{+}^{\mathrm{d}}$, anions $D_{-}^{\mathrm{d}}$ and cation-anion pairs $D_{ \pm}^{\mathrm{d}}$, which can be computed from MD simulations. ${ }^{28,32}$ The name "distinct" means it is cross-correlation between two different ions, even within the same species.

This leads to a decomposition of the Green-Kubo conductivity as

$$
\begin{gathered}
\sigma_{\mathrm{G}-\mathrm{K}}=q^{2} \rho \beta\left(D_{+}+D_{-}+D_{+}^{\mathrm{d}} / 2+D_{-}^{\mathrm{d}} / 2-D_{ \pm}^{\mathrm{d}}\right) \\
=\sigma_{\mathrm{N}-\mathrm{E}}+\sigma_{+}^{\mathrm{d}}+\sigma_{-}^{\mathrm{d}}+\sigma_{ \pm}^{\mathrm{d}}
\end{gathered}
$$

where $\sigma_{+}^{\mathrm{d}}, \sigma_{-}^{\mathrm{d}}$ and $\sigma_{ \pm}^{\mathrm{d}}$ are contributions to the ionic conductivity from the corresponding distinct diffusion coefficients. 

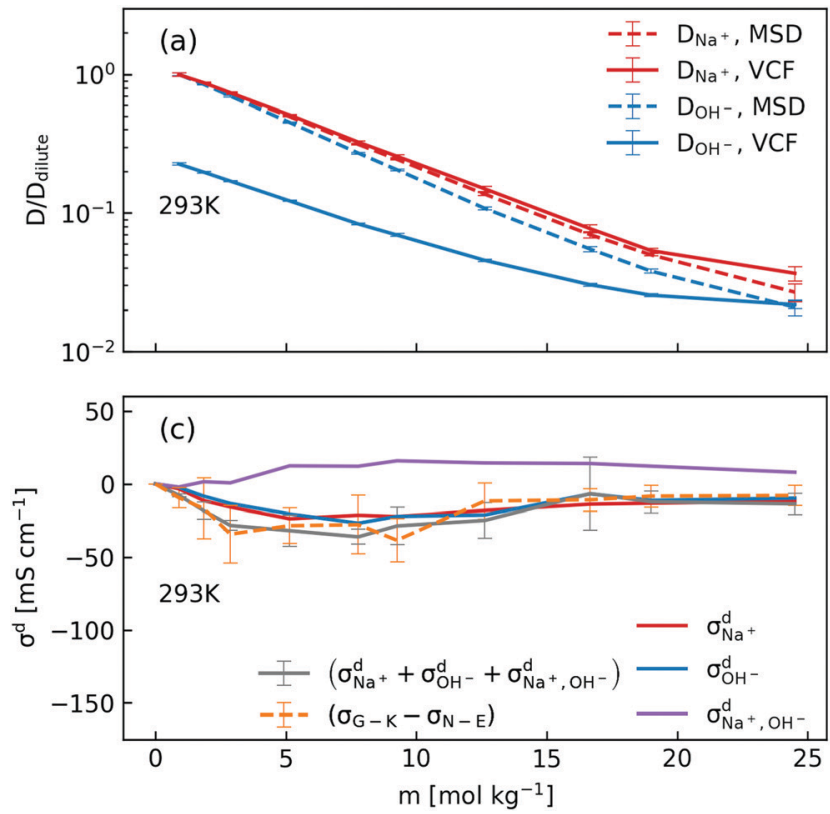
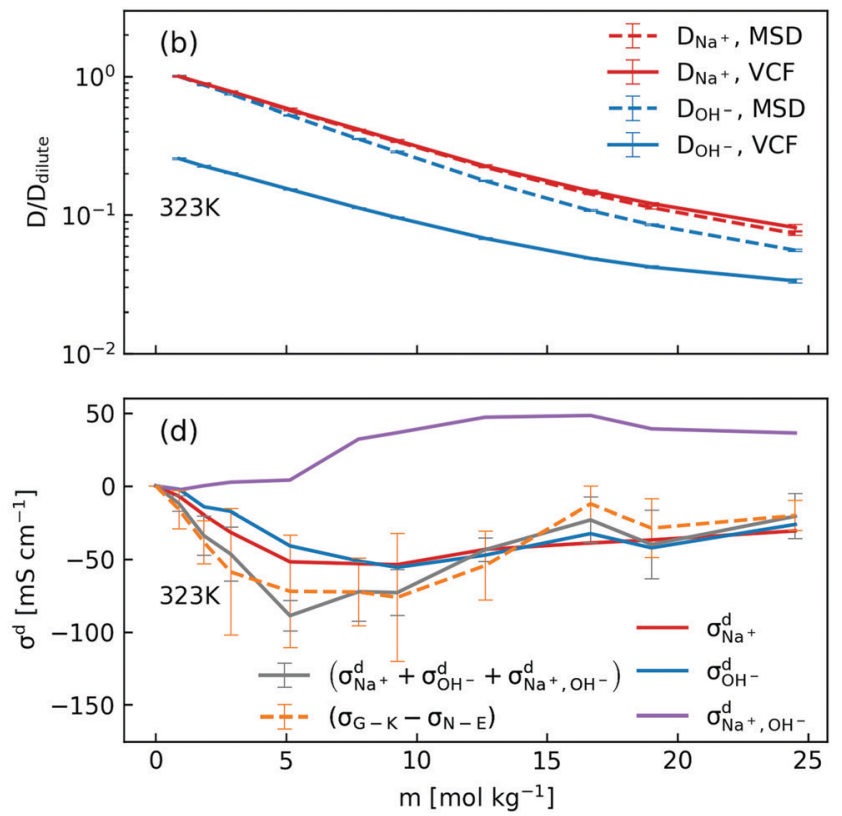

Fig. 2 (a) and (b) Scaled concentration-dependent self-diffusion coefficients of $\mathrm{Na}^{+}$and $\mathrm{OH}^{-}$at $293 \mathrm{~K}$ and $323 \mathrm{~K}$ obtained from VCF (eqn (2)) and from MSD (eqn (3)), where the self-diffusion coefficients were scaled by the corresponding value in the dilute solution; (c) and (d) contributions of distinct diffusion coefficients to the ionic conductivity at $293 \mathrm{~K}$ and $323 \mathrm{~K}$ as shown in eqn (7) and calculated from VCFs. Detailed definition of distinct diffusion coefficients can be found in ref. 28 and 32. Note that $\left(\sigma_{\mathrm{G}-\mathrm{K}}-\sigma_{\mathrm{N}-\mathrm{E}}\right)$ were obtained from Fig. 1a for the purpose of comparison.

After elaborating on the difference between the NernstEinstein conductivity and the Green-Kubo conductivity, we are now ready to compare the ionic conductivity calculated from MD simulations to those measured in experiments. The results are shown in Fig. 1. In Fig. 1a, the calculated ionic conductivities from MD simulations agree well with conductivity measurements, especially at $293 \mathrm{~K}$. Considering that the $\mathrm{NNP}^{19}$ was generated using only DFT calculations at the dispersion-corrected GGA level as the reference, this agreement is quite encouraging. Further improvement might be achieved with higher-level functionals. ${ }^{33}$ Note that we have neglected the finite-size correction $^{34}$ to the Nernst-Einstein conductivity showed in Fig. 1a, because of the relatively large simulation box that we used and the high viscosity of concentrated $\mathrm{NaOH}$ solutions (see Fig. S1 in the ESI $\dagger$ ).

When inspecting the simulation results at both $293 \mathrm{~K}$ and $323 \mathrm{~K}$ (Fig. 1), one can see that the Nernst-Einstein conductivity is always larger than the Green-Kubo conductivity, as expected. However, there are several interesting observations specific to $\mathrm{NaOH}$ solutions. First, the absolute difference between the Nernst-Einstein conductivity and the Green-Kubo conductivity becomes smaller at higher concentrations which is counterintuitive. Second, the difference is in general larger at higher temperature $(323 \mathrm{~K})$ than at lower temperature $(293 \mathrm{~K})$. Third, near the solubility limit $(25 \mathrm{~m})$ at room-temperature, the ionic conductivity at $323 \mathrm{~K}$ is still substantial while at $293 \mathrm{~K}$ it becomes quite small.

One way to rationalize these observations is to consider ion-pairing. For this reason, we calculated the coordination number of $\mathrm{OH}^{-}$around $\mathrm{Na}^{+}$ions as well the residence time of $\mathrm{Na}^{+}-\mathrm{OH}^{-}$pairs. The coordination number $(\mathrm{CN})$ was calculated by integrating the radial distribution function to its first minimum and the residence time $\tau$ was calculated by following the stable states picture formalism. ${ }^{35}$ As is described in ref. 20, a time correlation function $C(t)$ was calculated to give the probability that a "stable" hydroxide ion at time $t$ does not escape the first coordination shell of $\mathrm{Na}^{+}$through either ligand exchange or proton transfer within the interval $t_{0}$ and $t_{0}+t$. $C(t)$ was then fitted to a biexponentially decaying function to extract the residence time. As shown in Fig. 1b, the number of $\mathrm{OH}^{-}$coordinating $\mathrm{Na}^{+}$at both $293 \mathrm{~K}$ and $323 \mathrm{~K}$ can exceed one near the room-temperature solubility limit. However, the residence time at $293 \mathrm{~K}$ increases much more rapidly with the concentration than that at $323 \mathrm{~K}$. Based on these observations, one may speculate that the effect of ion-pairing on the ionic conductivity would be stronger at $293 \mathrm{~K}$.

In order to dissect the contributions of proton transfer reactions and cross-correlated ion motions, we exploited the fact that proton transfer contributes to the mean squared displacement (MSD, eqn (3)) but not to the corresponding velocity correlation function (VCF, eqn (2)). As shown in Fig. $2 \mathrm{a}$ and $\mathrm{b}$, the scaled self-diffusion coefficients of $\mathrm{Na}^{+}$are the same regardless whether they are computed from MSD or from VCF. In contrast, the scaled self-diffusion coefficients of $\mathrm{OH}^{-}$computed from MSD and VCF are different and their difference quantifies the contribution from proton transfer reactions. Comparing to the case at $323 \mathrm{~K}$, one can clearly see that the proton transfer contribution to the self-diffusion coefficient of $\mathrm{OH}^{-}$becomes negligible at $293 \mathrm{~K}$ in concentrated $\mathrm{NaOH}$ solutions. This is the main reason why the ionic conductivity near the room-temperature solubility limit at $323 \mathrm{~K}$ is still significant while at $293 \mathrm{~K}$ it becomes severely diminished. 
We then used the same technique to evaluate the contributions of the distinct diffusion coefficients to the ionic conductivity from VCFs, as shown in Fig. 2c and d. It is found that $\sigma_{\mathrm{Na}^{+}, \mathrm{OH}^{-}}^{\mathrm{d}}$ at both $293 \mathrm{~K}$ and $323 \mathrm{~K}$ are positive instead of negative as a simple picture of ion-pairing would suggest, similar to what was seen in ionic liquids. ${ }^{32}$ Further, $\sigma_{\mathrm{Na}^{+}, \mathrm{OH}^{-}}^{\mathrm{d}}$ is much more positive at $323 \mathrm{~K}$ than that at $293 \mathrm{~K}$. These suggest that in spite of the existence of ion-pairs in concentrated $\mathrm{NaOH}$ solutions, the effect of ion-pairing on the ionic conductivity should not be taken for granted.

When comparing the sum of $\sigma_{\mathrm{Na}^{+}}^{\mathrm{d}}, \sigma_{\mathrm{OH}^{-}}^{\mathrm{d}}$ and $\sigma_{\mathrm{Na}^{+}, \mathrm{OH}^{-}}^{\mathrm{d}}$ calculated from VCFs to the absolute difference between $\sigma_{\mathrm{G}-\mathrm{K}}$ and $\sigma_{\mathrm{N}-\mathrm{E}}$ calculated from the MSD, one can see a good agreement within the statistical error. Note that $\left(\sigma_{\mathrm{G}-\mathrm{K}}-\sigma_{\mathrm{N}-\mathrm{E}}\right)$ has a larger error bar than its counterpart $\left(\sigma_{\mathrm{Na}^{+}}^{\mathrm{d}}+\sigma_{\mathrm{OH}^{-}}^{\mathrm{d}}+\sigma_{\mathrm{Na}^{+}, \mathrm{OH}^{-}}^{\mathrm{d}}\right)$, because the former is the difference of two large numbers (Fig. 1a). The agreement between these two quantities means that the cross-correlated ion motions are hydrodynamic in nature and not determined by proton transfer reactions in $\mathrm{NaOH}$ solutions. We suspect that the counter-intuitive observation that $\left(\sigma_{\mathrm{G}-\mathrm{K}}-\sigma_{\mathrm{N}-\mathrm{E}}\right)$ becomes smaller at high concentration is related to the rapid increment of the viscosity in $\mathrm{NaOH}$ solutions (see Fig. S2 in the ESI $\dagger$ ). Since the viscosity decreases at elevated temperatures, this may also explain a larger difference between the Nernst-Einstein conductivity and the Green-Kubo conductivity at $323 \mathrm{~K}$ as seen in Fig. 1a. Nevertheless, future investigations are needed to identify the factors affecting the cross-correlated ion motions and subsequent deviations from the Nernst-Einstein relation. ${ }^{36}$

\section{Conflicts of interest}

There are no conflicts to declare.

\section{Acknowledgements}

CZ thanks Uppsala University (UU) for a start-up grant and funding from the Swedish National Strategic e-Science programme eSSENCE. MH acknowledges funding from the European Union's Horizon 2020 research and innovation programme under grant agreement No. 798129. KH acknowledges funding from the Swedish Research Council (VR). JB acknowledges the DFG for a DFG Heisenberg professorship (Be3264/11-2, project 329898176). The simulations were performed on the resources provided by the Swedish National Infrastructure for Computing (SNIC) at NSC.

\section{Notes and references}

1 G. Merle, M. Wessling and K. Nijmeijer, J. Membr. Sci., 2011, 377, 1-35.

2 A. R. Mainar, O. Leonet, M. Bengoechea, I. Boyano, I. de Meatza, A. Kvasha, A. Guerfi and J. A. Blázquez, Int. J. Energy Res., 2016, 40, 1032-1049.
3 E. Hückel, Z. Elektrochem. Angew. Phys. Chem., 1928, 34, 546-562.

4 N. Agmon, Chem. Phys. Lett., 1995, 244, 456-462.

5 M. E. Tuckerman, K. Laasonen, M. Sprik and M. Parrinello, J. Phys.: Condens. Matter, 1994, 6, A93-A100.

6 M. E. Tuckerman, D. Marx and M. Parrinello, Nature, 2002, 417, 925-929.

7 D. Marx, A. Chandra and M. E. Tuckerman, Chem. Rev., 2010, 110, 2174-2216.

8 A. Hassanali, M. K. Prakash, H. Eshet and M. Parrinello, Proc. Natl. Acad. Sci. U. S. A., 2011, 108, 20410-20415.

9 M. Chen, L. Zheng, B. Santra, H.-Y. Ko, R. A. D. Jr, M. L. Klein, R. Car and X. Wu, Nat. Chem., 2018, 10, 413-419.

10 T. J. F. Day, U. W. Schmitt and G. A. Voth, J. Am. Chem. Soc., 2000, 122, 12027-12028.

11 S. T. Roberts, P. B. Petersen, K. Ramasesha, A. Tokmakoff, I. S. Ufimtsev and T. J. Martinez, Proc. Natl. Acad. Sci. U. S. A., 2009, 106, 15154-15159.

12 R. Biswas, Y.-L. S. Tse, A. Tokmakoff and G. A. Voth, J. Phys. Chem. B, 2016, 120, 1793-1804.

13 Y. Marcus and G. Hefter, Chem. Rev., 2006, 106, 4585-4621. 14 S. V. Guaitolini and J. F. Fardin, in Advances in Renewable Energies and Power Technologies, ed. I. Yahyaoui, Elsevier, 2018, pp. 123-150.

15 V. Rozsa, D. Pan, F. Giberti and G. Galli, Proc. Natl. Acad. Sci. U. S. A., 2018, 115, 6952-6957.

16 C. Zhang, J. Hutter and M. Sprik, J. Phys. Chem. Lett., 2019, 10, 3871-3876.

17 W. Zhang and A. C. T. van Duin, J. Phys. Chem. C, 2015, 119, 27727-27736.

18 J. Behler and M. Parrinello, Phys. Rev. Lett., 2007, 98, 146401.

19 M. Hellström and J. Behler, J. Phys. Chem. Lett., 2016, 7, 3302-3306.

20 M. Hellström and J. Behler, J. Phys. Chem. B, 2017, 121, 4184-4190.

21 M. Hellström, M. Ceriotti and J. Behler, J. Phys. Chem. B, 2018, 122, 10158-10171.

22 S. Plimpton, J. Comput. Phys., 1995, 117, 1-19.

23 A. Singraber, J. Behler and C. Dellago, J. Chem. Theory Comput., 2019, 15, 1827-1840.

24 R. H. Perry, D. W. Green and J. O. Maloney, Perry's Chemical engineers' handbook, McGraw-Hill, New York, 6th edn, 1984.

25 G. Bussi, D. Donadio and M. Parrinello, J. Chem. Phys., 2007, 126, 014101.

26 H. W. Kuhn, Nav. Res. Logist. Q., 1955, 2, 83-97.

27 J. P. Hansen and I. R. McDonald, Phys. Rev. A: At., Mol., Opt. Phys., 1975, 11, 2111-2123.

28 E. C. Zhong and H. L. Friedman, J. Phys. Chem., 1988, 92, 1685-1692.

29 S. Chowdhuri and A. Chandra, J. Chem. Phys., 2001, 115, 3732-3741.

30 M. P. Allen and D. J. Tildesley, Computer simulation of liquids, Oxford university press, 2017.

31 J.-M. Caillol, J. Chem. Phys., 1994, 101, 6080-6090. 
32 H. K. Kashyap, H. V. R. Annapureddy, F. O. Raineri and C. J. Margulis, J. Phys. Chem. B, 2011, 115, 13212-13221.

33 T. Duignan, G. K. Schenter, J. Fulton, T. Huthwelker, M. Balasubramanian, M. Galib, M. D. Baer, J. Wilhelm, J. Hutter, M. D. Ben, X. S. Zhao and C. J. Mundy, ChemRxiv, 2019, DOI: 10.26434/chemrxiv.7466426.v2.
34 I.-C. Yeh and G. Hummer, J. Phys. Chem. B, 2004, 108, 15873-15879.

35 D. Laage and J. T. Hynes, J. Phys. Chem. B, 2008, 112, 7697-7701.

36 Y. Shao, K. Shigenobu, M. Watanabe and C. Zhang, ChemRxiv, 2019, DOI: 10.26434/chemrxiv.8217152.v2. 